\title{
ANGULAR MOMENTUM TRANSPORT AND MAGNETIC FIELDS IN THE SOLAR INTERIOR
}

\author{
H.C. SPRUIT \\ Max Planck Institut für Physik und Astrophysik \\ Karl-Schwarzschildstr. 1,8046 Garching West Germany
}

\begin{abstract}
The possible mechanisms of angular momentum transport in convectively stable regions of a star are reviewed, with emphasis on transport by magnetic torques. The strength and configuration of the field in such layers is quite uncertain, because it is not known if the field can reach a dynamically stable configuration. A lower limit to the field strength is obtained by assuming that the field is always dynamically unstable, and decaying at the (rotation modified) dynamical time scale. The present field in the sun would then be of the order $1 \mathrm{G}$, with poloidal and toroidal components of similar strength. The differential rotation in the core, if due only to the solar wind torque, would be very small for this field strength, and instead would more likely be governed by magnetic coulpling to the differential rotation of the convection zone. If small scale hydrodynamic transport mechanisms are present, their properties would also be influenced by a field of this strength.
\end{abstract}

\section{Introduction}

The internal rotation of the sun in the convectively stable core is remarkably uniform (Duvall and Harvey, 1984; Duvall, this volume; Brown et al. 1989). Though the lowest degree p-modes $(1=1,2,3)$ indicate a possible rise in rotation speed inside $r=0.3 R_{\odot}$ (Duvall and Harvey, 1984), the statistical significance of this rise is not large. In any case, the present limits on the differential rotation already put rather stringent constraints on proposed mechanisms for angular momentum transport in the core. These mechanisms come in roughly four flavors, (i) turbulent stress associated with shear instabilities generated by the differential rotation itself, (ii) meridional circulations due to the rotational deformation of the star or the external torque, (iii) angular momentum transport by waves generated by the convection zone, and (iv) magnetic stresses. These mechanisms are each discussed briefly below. The upshot will be that magnetic fields are arguably the most effective in enforcing uniform rotation, but that they are also the least amenable to quantitative theory at this time.

\subsection{A Clue: The Lithium Depletion}

The solar Lithium abundance is a factor of 100 below its primordial value. Stars like the sun

G. Berthomieu and M. Cribier (eds.), Inside the Sun, 415-423.

C) 1990 Kluwer Academic Publishers. Printed in the Netherlands. 
show a gradual decline of Lithium abundance with age (Soderblom 1988). This is believed to be due to a slow mixing process in outer parts of the radiative core, which transports the Lithium from the convection zone down until it reaches its burning temperature $(\approx$ $2.810^{6} \mathrm{~K}$ ) (Baglin, Morel, Schatzman, 1985; Vauclair, 1988). Such a mixing process is also likely to transport angular momentum and the relative efficiency of mixing and angular momentum transport depends on the mechanism. Hence if the low internal rotation and the Lithium depletion are the consequence of a single process, combining these observations would give a clue on its nature. Small scale turbulence for example is likely to have a turbulent diffusivity and turbulent viscosity of the same order of magnitude. Magnetic fields on the other hand can in principle exert torques without causing any mixing.

\section{Hydrodynamics}

The solar wind exerts, through its coupling with the atmospheric magnetic fields generated by the convection zone, a torque (Schatzman, 1962) that would spin the sun down on a time scale (Pizzo et al. 1984) similar to its present age. The radiative layers below the convection zone will not take part in this spindown unless there is a mechanism that couples them to the convection zone.

\subsection{SHEAR INSTABILITIES}

The radial gradient of rotation rate that builds up during spindown may become unstable to various shear instabilities. The thermal gradient is strongly stabilizing (the buoyancy frequency being much larger than the rotation rate). On sufficiently small scales the high thermal conductivity due to radiation (low Prandtl number) cancels this stabilizing effect. Another stabilizing effect is the inward increase of the mean weight per particle (Helium inside, Hydrogen outside). This stabilization remains effective also on small scales. An approximate condition for the onset of shear instability taking these factors into account has been derived by Zahn $(1974,1983)$. In addition, a number of more subtle instabilities exists (for reviews see Zahn, 1983; Spruit, 1987a; Zahn, this volume). A minimum rotation curve for the present sun, assuming the minimum gradient needed to keep at least one of the instabilities acting, was derived by Spruit et al. (1983). It is not compatible with the observed p-mode splittings (Duvall and Harvey 1984), which require significantly lower rotation rates. Thus, the known shear instabilities can not account for the inferred internal torques in the core. Tassoul (1989), referring to conditions in the earth oceans, has argued that the turbulent motions could be highly anisotropic (due to the strong influence of the stabilizing buoyancy force) and that the measured diffusivity for momentum in these cases is significantly higher than that for composition. In the cases referred to, wave motions (internal gravity waves) make up a sigificant fraction of the 'turbulence', and the transport properties of these waves are indeed quite different from isotropic turbulence. To make a valid comparison with the solar interior one has to establish what sources of wave motion exist in the sun, and how their transport properties differ in the sun's low Prandtl number climate. 


\subsection{Circulations}

The deformation of the sun due to its rotation induces a slow meridional circulation, the Eddington-Sweet-Vogt circulation. It is driven by a small difference in heat flux between the pole and the equator (e.g. Zahn, 1983). It has been too weak over most of the sun's history to transport much angular momentum. It may perhaps produce a low level of turbulence through Zahn's scenario (see Zahn, this volume), enough to be relevant for the Lithium depletion. Another circulation is an Ekman flow driven by a difference in rotation rate between the convection zone and the interior. It has been studied in detail (Benton and Loper, 1970; Benton and Clark, 1974; Sakurai, 1975; Osaki, 1982). Its effect is negligible except in a very thin layer below the convection zone.

\subsection{Waves}

The sun is traversed by sound waves excited in the top of the convection zone. In addition, the unsteady nature of the convective flow at the base of the convection zone is expected to generate internal gravity waves in the core, though such waves have not been measured yet. Waves can exert torques by being refracted or absorbed at some distance from the place they were excited. If the wave field is excited asymmetrically with respect to the direction of rotation (eastward moving waves having slightly different amplitudes or propagation speeds than westward waves for example), they can exert a selective spinup or spindown torque at the place where they dissipate. But even a symmetrically excited wave field will exert a 'viscous' stress by exchanging momentum between parts of the shearing flow (the analogy of wave packets with particles holds here). A related problem in astrophysics is that of the tidal interaction in a binary (Zahn, 1977, Goldreich and Nicholson, 1989 and references therein). Press (1981) has stressed that internal gravity waves generated at the base of the convection zone may be quite important. If $\omega_{0}$ is the frequency of the dominant convective eddy near the base, $N$ a typical buoyancy frequency in the core just below the convection zone, one estimates a wave energy flux into the core which is a fraction $\omega_{0} / N$ of the solar energy flux itself. This is of the order $10^{-3}\left(\omega_{0} \sim 10^{-6} s^{-1}, N \sim 10^{-3} s^{-1}\right)$. Most of this flux is in the form of waves with a very small vertical length scale however, which dissipate by radiative damping within a very short distance below the convection zone. The interesting waves are those generated by the high frequency, large length corner in the spectrum of motions of the convection zone. The energy in this corner is poorly estimated by current theories of the convection zone. Numerical simulations of wave excitation by the convection zone seem in order ( $c f$. Hurlburt et al. 1986).

\section{Magnetic fields}

The role of magnetic fields is much harder to quantify than any of the above processes, because a number of fairly basic things are unknown. On the other hand, magnetic fields may well be of central importance, at least for the angular momentum transport in the core. 
From observations at the surface, estimates of the field strength in the convection zone can be made. If most of the field resides near the base of the convection zone during the activity cycle (as seems likely for reasons of stability, Moreno Insertis 1986, Schmitt et al. 1984, van Ballegooijen, 1982, Spruit and Roberts, 1983), it would plausibly be of the order $10000 \mathrm{G}$, covering a depth of the order $1000-10000 \mathrm{~km}$. The field strength in the core is essentially unknown, with upper limits of the order $10^{7}$ placed by helioseismology measurements (Dziembowski, this volume). Increasing with its strength, the field has effects of decreasing subtlety. Order of magnitudes can be made based on time scales.

\subsection{TIME SCALES}

The dynamic time scale of the sun (the sound crossing time or the the time for a sattelite to orbit once around its surface) is of the order $t_{d}=(R / g)^{1 / 2} \sim 210^{3} \mathrm{~s}$, where $R=710^{10}$ $\mathrm{cm}$ is the solar radius and $g=2.710^{4} \mathrm{~cm} \mathrm{~s}^{-2}$ its surface gravity. Its rotational period $T=2 \pi / \Omega$ is $210^{6} \mathrm{~s}(25 \mathrm{~d})$. The spindown time scale due to the solar wind torque is of the same order as the age of the sun, at any point in time; hence presently of the order $t_{s}=10^{17} \mathrm{~s}$.

These time scales can be compared with the magnetic time scale, which is the time it takes an Alfvén wave to cross the core:

$$
t_{B}=R / v_{A}=\sqrt{4 \pi \rho} R / B \quad \sim 1.510^{10} / B \quad \mathrm{~s}
$$

(with a mean density $\rho \sim 1$ for the core). This is the time scale on which the field can communicate forces across the core. It is also the time scale on which the field will evolve, if it is dynamically unstable. If

$$
t_{B} \sim t_{d} \quad \text { or } \quad B=B_{1} \sim(8 \pi P)^{1 / 2} \quad\left(\sim 10^{8} \mathrm{G}\right)
$$

the field has a significant influence on the shape of the star. From upper limits on distortions measured helioseismologically, such high fields can be excluded. When

$$
t_{B} \sim T \text { or } \quad B=B_{2} \sim \Omega R \sqrt{4 \pi \rho} \quad\left(\sim 310^{5} \mathrm{G}\right)
$$

the influence of the field on the p-mode frequencies is of the same order as the rotational splitting of these modes. This kind of field should be measurable helioseismologically. A field of the order

$$
t_{B} \sim\left(t_{s} T\right)^{1 / 2} \quad \text { or } \quad B=B_{3} \sim\left(\frac{\Omega}{t_{s}}\right)^{1 / 2} R \sqrt{4 \pi \rho} \quad(\sim 1 \mathrm{G})
$$

is of special importance; it is the field that is just large enough to keep the core corotating with the surface, under the influence of the solar wind torque. When

$$
t_{B} \sim t_{s} \quad \text { or } \quad B=B_{4} \sim \frac{R}{t_{s}} \sqrt{4 \pi \rho} \quad\left(\sim 10^{-6} \mathrm{G}\right)
$$

the field redistributes angular momentum on a time scale equal to the age of the sun; fields lower than this are therefore not important in the present context. The values in brackets in these expressions are for the present sun. 


\subsection{Strength of the initial Field}

In view of the limited information observations provide on the present field strength in the core, it is useful to consider the the field as the sun was born, and speculate a bit on how strong it could have been. Star formation, at least in its later stages, proceeds through accreting gas from a disk surrounding the protostar. These disks collect gas from distances very large compared with the size of the star; the field embedded in it at that distance gets compressed as it drifts in with the accreting gas. Near the star, this field is of a single polarity with respect to the disk plane at any point in time (history of the accretion process or statistics of the field in the original source of the gas determining which polarity). Support for the assumption of such a stable (over many orbital periods) field of one polarity comes from the observed jets of protostars, which are most naturally explained by magnetic acceleration (Blandford and Payne, 1982; Lovelace et al. 1987; Pudritz and Norman, 1983; Königl, 1989) in such a configuration. If the strength of the field caught in the gas at large distance exceeds a certain plausible minimum, the strength of the field near the protostellar surface is determined only by the local balance between the magnetic stress (pulling fields away from the star) and the accretion. By equating the magnetic curvature (the dominant stress component in this case) with the radial gas pressure gradient yields a field strength of the order

$$
B=\alpha^{-1 / 2}(G M)^{1 / 4} r^{-5 / 4} \dot{M}^{1 / 2} \quad \mathrm{G}
$$

where $G$ is the gravitation constant, $M$ the mass of the protostar, $\alpha$ the disk viscosity parameter, $r$ the radial distance, and $\dot{M}$ the mass acretion rate (all $\mathrm{cgs}$ ). This holds for a radially selfsimilar disk ( $c f$. Blandford and Payne, 1982). For a protostar of $1 M_{\odot}$, accreting $10^{-5} M_{\odot} \mathrm{yr}^{-1}, \alpha=0.1$ this yields several hundred $\mathrm{G}$ at the stellar surface $r=10^{11}$. So an ordered poloidal field of significant strength is accreted onto the star.

\subsection{Simple MOdels FOR DIFFERENTIAL ROTATION WITH B-FIELDS}

3.3.1 Axisymmetric field As a model problem, consider an axisymmetric (about the rotation axis) initially poloidal field $\mathbf{B}_{p}$ (field lines in meridional planes) (Mestel and Weiss, 1987 and references therein, Rädler, 1986). Assume the initial field to be weak (but larger than $B_{4}$ above). The problem is to find how the differential rotation $\Omega(r, \theta)$ evolves in time. An azimuthal field component develops due to stretching of the field lines by the differential rotation. If $\Delta \Omega$ is a measure of the difference in rotation rate occurring along a particular field line, this component initially grows as

$$
B_{\phi} \sim B_{p} t \Delta \Omega .
$$

The model can be calculated only if it is further assumed that the poloidal field component remains unchanged during the evolution. This way one avoids, in particular, the difficult issue of the stability of the field. The magnetic stress due to the distortion of the field then exerts a torque $B_{\phi} B_{p} / 4 \pi$ which increases linearly with the azimuthal displacement $t \Delta \Omega$. 
As a result the motion on each of the axisymmetric magnetic surfaces corresponding to a particular field line of $\mathrm{B}_{p}$ is that of a harmonic oscillator. The period of oscillation is the Alfvén travel time along the field line.

3.3.2 Phase mixing. In the absence of dissipative processes the oscillation would go on indefinitely. Since each of the magnetic surfaces oscillates completely independently of the others however, and the Alfvén travel times vary between the surfaces, the oscillations on the surfaces will get increasingly out of phase with each other. The length scale across the surfaces on which the phase varies decreases as $1 / t$, and after a finite time magnetic diffusion (the fastest relevant dissipative mechanism) becomes effective and damps the motion. This process is called damping by phase mixing, and has been studied in the coronal context (Ionson, 1978; Heyvaerts and Priest, 1983). Calculations in the present context have been made by Roxburgh (private communication). The net effect is that an initial differential rotation, not driven by an external torque, will dissipate on a time scale that is some multiple of the initial Alfvén travel time, depending only weakly on the value of the diffusivity.

When an external torque is applied, transients are set up that damp by the same mechanism. After this, a configuration results in which the azimuthal field $B_{\phi}$ has grown to just that amplitude at which the magnetic stress transmits the external torque on the same time scale as the star spins down. For the present sun, this means that the average of $\left(B_{r} B_{\phi}\right)^{1 / 2}$ would be of the order $1 \mathrm{G}$. The total field strength $\left(B_{p}^{2}+B_{\phi}^{2}\right)^{1 / 2}$ is minimized, for a given $B_{p} B_{\phi}$, when $B_{\phi}$ and $B_{p}$ are of the same order of magnitude. Thus the value Eq. (4) is the minimum field strength required to transmit the external torque, and at this minimum the azimuthal and poloidal components are of similar magnitude.

It must be stressed that this is a simplistic picture, since it does not take into account that dynamical, nonaxisymmetric instabilities may drastically alter the field configuration at some stage of the process.

3.3.3 Closed field lines The above implicitly assumes that all of the core is connected with the surface by a field line. This is not necessarily the case, there may be regions (tori) with closed field lines. If axisymmetric, these could rotate at their own rate, hardly affected by the external torques (e.g. Mestel and Weiss, 1987).

3.3.4 Nonaxisymmetric fields Nonaxisymmetric cases have effectively been studied only in the kinematic approximation; that is the Lorentz force is ignored altogether. Hence such models address the evolution of the field but not that of the differential rotation. Rädler (1986) (see also Zeldovich et al. 1983) shows that the nonaxisymmetric component of the field is 'wrapped up' on the differential rotation time scale, so again the length scale on which the field changes direction decreases as $1 / t$, as in the phase mixing process addressed above. Magnetic diffusion then destroys the nonaxisymmetric part of the field on a time scale of a few differential rotations, depending only weakly on the value of the diffusivity, and an axisymmetric field survives.

\subsection{STABILITY OF FIELDS}

Dynamical instability of the field (that is, ignoring dissipative processes), is a major concern, 
since it changes the configuration on an Alfvén time scale. For the field strength that just suffices to transmit the present solar wind torque to the core ( $B_{3}$ in section 3.1$)$, this time scale is or the order $10^{4} \mathrm{yr}$, much shorter than the time scale on which we want the field to to its work (but see the modification to the instability time scale by rotation, below). Are fields likely to be dynamically unstable? It is known (Tayler, 1980, van Assche et al. 1982) that all purely poloidal fields in stars, as well as all purely toroidal fields are dynamically unstable. Stable fields at least require a combination of toroidal and poloidal components of similar strength (Mestel, 1984). No example of a dynamically stable equilibrium field in a star is known (but they are not so easy to construct). One might be tempted to guess that all fields are dynamically unstable in stars. This is probably incorrect though, since (Moffat, private communication) in the absence of diffusion there is a conservation property that limits the amount of energy an equilibrium can release by an instability. If $\mathbf{A}$ is the vector potential of $\mathbf{B}$, the integral of the magnetic helicity $\mathbf{B} \cdot \mathbf{A}$ over the field configuration is conserved for frozen in fields (Woltjer, 1958; see Berger, 1986). In mixed poloidal-toroidal fields the net helicity is generically nonzero. Since zero magnetic energy means zero net helicity, there is for each mixed poloidal- toroidal field a nonzero minimum energy configuration. Whether such configurations can develop in a star, and whether they will remain stable when magnetic diffusion is included is another question (Mestel, 1984 and references therein).

\subsection{A lower limit to the PRESEnt field StRength in the CORE}

The field strengths to be found at the bottom line of this text are such that the Alfvén travel time is much larger than the rotation period. In this circumstance, the coriolis force has a strong influence on the growth rate of an instability. Motions parallel to the rotation axis are not affected by the coriolis force, but unstable displacements can generally not be constructed out of such motions alone. As a result, the inclusion of rotation usually reduces the growth rates by a factor $t_{B} \Omega$ compared with the nonrotating case (see Pitts and Tayler, 1985 for examples). Dynamically unstable fields therefore reconfigure on a timescale

$$
t_{\text {inst }}=t_{B}^{2} \Omega . \quad\left(\Omega t_{B} \gg 1\right) .
$$

With this, we can do a consistency check on the initial field strength assumed in section 3.2. Inserting $B$ from Eq. (6) we find for the initial instability time scale

$$
t_{i n s t} 0 \sim \alpha \frac{\Omega}{\Omega_{m}} \frac{M}{\dot{M}}
$$

where $\Omega_{m}$ is the maximum rotation rate of the protostar, the Kepler rotation rate at its surface. Since the field is built up by accretion on the time scale $t_{a c c}=M / \dot{M}$, the value given by Eq. (6) can be sustained against possible dynamic instability if the rotation rate is near its maximum. The stellar wind torque on the young star rapidly reduces its rotation rate, and the growth rate of dynamic instabilities increases. We can now estimate a lower limit on the field strength in the star by assuming that the field always is dynamically unstable. Of course, the field may well get stuck at some point in time into a stable configuration, but then the field, limited now by slower diffusive processes, will only be higher than we are estimating here. If the instability time scale is shorter than the spindown time scale, the 
field will get smaller; if it is longer, the rotation rate decreases until by Eq. (8) the instability time scale has decreased to match the spindown time scale again (Spruit, 1987b). Thus for a wide range of conditions, the field attains a typical value that is largely independent of the initial conditions, and decreases in time such that its dynamical instability time is the same as the spindown time of the star. The field value that follows from the condition $t_{\text {inst }}=t_{s}$ is just $B_{3}$ given in section 3.1. As discussed, it is the field strength that is just sufficient to transmit the stellar wind torque to the core, and the azimuthal field component is of the same order as the poloidal component.

At this strength, the magnetic energy density in the field is of the same order, for the present sun, as the kinetic energy in the turbulent motions envisaged in some of the hydrodynamic transport schemes (such as Zahn's scenario). Since our estimate is a lower limit, this shows it may not be realistic to neglect the magnetic field, even in purely hydrodynamic transport schemes.

For the field Eq. (4), the time scale for transmitting torques through the core is small compared with the spindown time (but long compared with the rotation period), by a factor $\sim 10^{6}$. Hence the level of differential rotation in the core, if due only to the processes described, should be quite small. (But there are obvious other processes, such as magnetic coupling to the differentially rotating convection zone, that will enforce some level of differential rotation).

Concluding, by assuming that the primordial field is dynamically unstable at all times during the spindown of the star, we have estimated a mimimum field strength to be expected in the core of the present sun, of the order of $1 \mathrm{G}$.

\section{References}

Baglin, A., Morel, P.J., Schatzman, E. 1985, Astron. Astrophys., 149, 309

Berger, M.A. 1986, Geophys. Astrophys. Fluid Dyn., 34, 256

Blandford, R.D., Payne, D.G. 1982, Mon. Not. R. astron. Soc., 199, 883

Benton, E.R., Loper, D.E. 1970, J. Fluid Mech., 43, 75

Benton, E.R., Clark, D.E. 1974, Ann. Rev. Fluid Mech. 6, 257

Brown, T.M. Christensen-Dalsgaard, J., Dziembowski, W.A., Goode, P., Gough, D.o., Morrow, C.A. 1989. Astrophys., J., 343526

Duvall, T.L. Jr., Harvey, J.W. 1984, Nature, 310, 19

Goldreich, P., Nicholson, P. 1989, preprint

Heyvaerts, J., Priest, E.R. 1983, Astron. Astrophys., 117, 220

Hurlburt, N.E., Toomre J., Massaguer, J.M. 1986, Astrophys., J., 311, 563

Ionson, J.A. 1978, Astrophys., J., 226, 650

Königl, A. 1989, Astrophys., J., 342, 208

Lovelace, R.V.E., Wang, J.C.L., Sulkanen, M.E. 1987, Astrophys., J., 315, 504

Mestel, L. 1984, Astron. Nachr. 305, 301

Mestel, L. Weiss, N.O. 1987, Mon. Not. R. astron. Soc., 226, 123

Moreno Insertis, F. 1986, Astron. Astrophys., 166, 291

Osaki, Y. 1982, Publ. Astr. Soc. Japan, 34, 257 
Pitts, E., Tayler, R.J. 1985, Mon. Not. R. astron. Soc., 216, 139

Pizzo, V., Schwenn, R., Marsch, E., Rosenbauer, H., Mühlhäuser, K.-H., Neubauer, F.M. 1983, Astrophys., J., 271, 335

Press, W.H. 1981. Astrophys., J., 245, 111

Pudritz, R.E., Norman, C.A. 1983, Astrophys., J., 274, 677

Rädler, K.-H., 1986, preprint

Sakurai, T. 1975, Mon. Not. R. astron. Soc., 171, 35

Schatzman, E., 1962, Ann. Astrophys., 222, 317

Schmitt, J.H.M.M., Rosner, R., Bohn, H.U. 1984, Astrophys., J., 282316

Soderblom, D. 1988 in The Impact of very high $S / N$ etc., eds. G. Cayrel de Strobel and M. Spite (IAU Symp. 132), Kluwer, Dordrecht, p381

Spruit, H.C. 1987a, in it Physical Processes on Comets, Stars and Active Galaxies, eds. W. Hillebrandt, E. Meyer-Hofmeister and H.-C. Thomas, Springer, Heidelberg, p78

Spruit, H.C., 1987b, in The Internal Solar Angular Velocity, eds. B.R. Durney and S. Sofia, Reidel, Dordrecht, p185

Spruit, H.C., Roberts, B. 1983, Nature, 304, 401

Spruit, H.C., Knobloch, E., Roxburgh, I.W. 1983, Nature, 304, 320

Tassoul, J.-L. 1989, Astron. Astrophys.,

Tayler, R.J., 1980, Mon. Not. R. astron. Soc., 191, 151

Vauclair, S. 1988, Astrophys., J., 335, 971

van Assche, W., Tayler, R.J. Goosens, M. 1982, Astron. Astrophys., 109, 166

van Ballegooijen, A.A. 1982. Astron. Astrophys., 113, 99

Woltjer, L. 1958, Proc. Natl. Acad. Sci. USA 44, 489

Zahn, J.-P. 1974, in Stellar Instability and Evolution, eds. P. Ledoux, A. Noels and R.W. Rogers, Reidel, Dordrecht, p185

Zahn, J.-P. 1983, in Astrophysical Processes in upper Main Sequence Stars, Geneva Observatory, Switzerland, p225

Zahn, J.-P. 1977, Astron. Astrophys., 57, 383

Zeldovich, Ya. B., Ruzmaikin, A.A., Sokoloff, D.D. 1983, Magnetic Fields in Astrophysics, Gordon And Breach, N.Y. 